\title{
Effects of Implementing Adaptive Support Ventilation in a Medical Intensive Care Unit
}

\author{
Chien-Wen Chen MD, Chin-Pyng Wu MD PhD, Yu-Ling Dai, Wann-Cherng Perng MD, \\ Chih-Feng Chian MD, Wen-Lin Su MD, and Yuh-Chin T Huang MD MHS
}

\begin{abstract}
BACKGROUND: Adaptive support ventilation (ASV) facilitates ventilator liberation in postoperative patients in surgical intensive care units (ICU). Whether ASV has similar benefits in patients with acute respiratory failure is unclear. METHODS: We conducted a pilot study in a medical ICU that manages approximately 600 mechanically ventilated patients a year. The ICU has one respiratory therapist who manages ventilators twice during the day shift (8:00 AM to 5:00 PM). No on-site respiratory therapist was present at night. We prospectively enrolled 79 patients mechanically ventilated for $\geq 24$ hours on pressure support of $\geq 15 \mathrm{~cm} \mathrm{H}_{2} \mathrm{O}$, with or without synchronized intermittent mandatory ventilation, $\mathrm{F}_{\mathrm{IO}_{2}} \leq 50 \%$, and PEEP $\leq 8 \mathrm{~cm} \mathrm{H} \mathrm{H}_{2} \mathrm{O}$. We switched the ventilation mode to ASV starting at a "\% MinVol" setting of 80-100\%. We defined spontaneous breathing trial (SBT) readiness as a frequency/tidal-volume ratio of $<105$ (breaths $/ \mathrm{min}$ )/L on pressure support of $\leq 8 \mathrm{~cm} \mathrm{H} \mathrm{H}_{2} \mathrm{O}$ and PEEP of $\leq 5 \mathrm{~cm} \mathrm{H} \mathrm{H}_{2} \mathrm{O}$ for at least $2 \mathrm{~h}$, and all spontaneous breaths. The T-piece SBT was considered successful if the frequency/tidal-volume ratio remained below 105 (breaths/min)/L for $30 \mathrm{~min}$, and we extubated after successful SBT. The control group consisted of 70 patients managed with conventional ventilation modes and a ventilator protocol during a 6-month period immediately before the ASV study period. RESULTS: Extubation was attempted in $73 \%$ of the patients in the ASV group, and $80 \%$ of the patients in the non-ASV group. The re-intubation rates in the ASV and non-ASV groups were 5\% and 7\%, respectively. In the ASV group, $20 \%$ of the patients achieved extubation readiness within 1 day, compared to $4 \%$ in the non-ASV group $(P=<.001)$. The median time from the enrollment to extubation readiness was 1 day for the ASV group and 3 days for the non-ASV group $(P=.055)$. Patients switched to ASV were more likely to be liberated from mechanical ventilation at 3 weeks $(P=.04)$. Multiple logistic regression analysis showed that, of the independent factors in the model, only ASV was associated with shorter time to extubation readiness $(P=.048$ via likelihood ratio test $)$. CONCLUSIONS: Extubation readiness may not be recognized in a timely manner in at least $15 \%$ of patients recovering from respiratory failure. ASV helps to identify these patients and may improve their weaning outcomes. Key words: mechanical ventilation; respiratory failure; closed-loop; automation; weaning. [Respir Care 2011;56(7):976-983. @ 2011 Daedalus Enterprises]
\end{abstract}

Drs Chen, Dai, Perng, Chian, and Su are affiliated with the Division of Pulmonary and Critical Care Medicine, Tri-Service General Hospital, Taipei, Taiwan. Dr Wu is affiliated with the Department of Medicine, Landseed Hospital, Taoyuan, Taiwan. Dr Huang is affiliated with the Department of Medicine, Duke University Medical Center, Durham, North Carolina.

This study was partly supported by a grant TSGH-C97-58 from TriService General Hospital, Taiwan. The authors have disclosed no conflicts of interest.
Correspondence: Chin-Pyng Wu MD PhD, Department of Thoracic Internal Medicine, Landseed Hospital, No 77, Kwang-Tai Road, Ping-Jen City, TaoYuan County, Taiwan. E-mail: wucp@ landseed.com.tw. Yuh-Chin T Huang MD, Department of Medicine, Duke University Medical Center, MHS, Box 102365, Durham NC 27710. E-mail: huang002@mc.duke.edu.

DOI: $10.4187 /$ respcare.00966 


\section{Introduction}

Several automated computerized systems for ventilatory management are now commercially available. These systems, by continuous monitoring and interventions, may be equivalent to or better at adapting to the patient's needs than conventional methods. ${ }^{1-3}$ One such system is adaptive support ventilation (ASV), which is available on the Galileo and G5 ventilators (Hamilton Medical, Rhazuns, Switzerland). ASV relies on closed-loop regulation of settings in response to changes in respiratory mechanics and spontaneous breathing ${ }^{4-6}$ Once a target minute volume $\left(\dot{\mathrm{V}}_{\mathrm{E}}\right)$ is entered by the clinician using a setting called $\% \mathrm{MinVol}$, ASV automatically determines a target tidal volume $\left(\mathrm{V}_{\mathrm{T}}\right)$ and respiratory rate combination based on the minimum work of breathing principle proposed by Otis et al. ${ }^{7}$ When the patient is on mandatory breaths, ASV can adjust both $\mathrm{V}_{\mathrm{T}}$ and respiratory rate to meet the target. If the patient is breathing spontaneously (pressure support breaths), ASV can adjust the inspiratory pressure.

There are several theoretical advantages of ASV. Because ASV can continuously reduce the inspiratory pressure as the patient and the lung mechanics improve, the process of ventilator liberation is not interrupted, even when staff support is limited. Clinical studies found that post-cardiac-surgery patients ventilated with ASV had either shorter or equivalent time to extubation..$^{8-12}$ ASV may also decrease inspiratory load, improve patient-ventilator interaction, ${ }^{13}$ and decrease the need to adjust ventilator settings. ${ }^{9}$ On the other hand, an inappropriate $\% \mathrm{MinVol}$ setting may adversely affect the progress of ventilator weaning. Too high a \% MinVol setting that excessively reduces the work of breathing and respiratory drive may lead to muscle atrophy and weakness, whereas too low a \% MinVol setting may increase patient anxiety and work of breathing.

In this pilot study, we prospectively evaluated the effects of ASV in patients who were recovering from acute respiratory failure in a medical ICU with a low respiratory therapist (RT) to patient ratio. We hypothesized that ASV would facilitate ventilator management by identifying patients ready for ventilator liberation and thus improve the probability of ventilator liberation. We compared patients prospectively managed with ASV to a control group of matched patients managed with conventional ventilation during the 6-month period immediately before the ASV study period.

\section{Methods}

This study was approved by the institutional review board of the Tri-Service General Hospital. Informed consent was obtained from all participants' next-of-kin prior to enrollment, in accordance with Taiwan law.

\section{Patients}

We prospectively screened patients admitted to the medical ICU of the Tri-Service General Hospital in Taipei, Taiwan, between December 1, 2006, and September 30, 2007. Eligible patients were those who were hemodynamically stable (ie, on no or low-dose vasopressors $[\leq 5 \mu \mathrm{g} /$ $\mathrm{kg} / \mathrm{min}$ of dopamine, or $\leq 0.05 \mu \mathrm{g} / \mathrm{kg} / \mathrm{min}$ of norepinephrine]) and who were ventilated with pressure support of $\geq 15 \mathrm{~cm} \mathrm{H}_{2} \mathrm{O}$, with or without synchronized intermittent mandatory ventilation (SIMV), and $\mathrm{S}_{\mathrm{aO}_{2}} \geq 90 \%$ on $\mathrm{F}_{\mathrm{IO}_{2}} \leq 50 \%$ and PEEP $\leq 8 \mathrm{~cm} \mathrm{H} \mathrm{H}_{2} \mathrm{O}$. We excluded patients with tracheostomy, patients who were judged by the attending physician to have less than 1 month to live, who had a do-not-resuscitate order, who had untreated cancer, or whose next-of-kin would not give consent for participation.

The matched control group was obtained by screening patients who were managed with conventional ventilation between April 1 and October 30, 2006. The RT respiratory $\log$, which was entered twice a day, was screened by an RT who was unaware of the study's hypothesis and outcome measures. Eligible patients were identified by the same inclusion and exclusion criteria as above. A separate approval was obtained from the institutional review board of Tri-Service General Hospital for the secondary analysis of existing data.

\section{Organization of the Medical ICU}

The medical ICU at Tri-Service General Hospital is a 16-bed closed unit with a full-time medical director, and manages approximately 600 mechanically ventilated patients per year. One full-time attending physician (intensivist) makes rounds in the morning with the ICU team, which consists of one fellow, 2 residents, and one RT, and reviews all events of the past night and sets a plan for the day, including ventilator management. The attending physician also conducts a more focused evening round each day.

\section{Adaptive Support Ventilation}

ASV is a volume-targeted, closed-loop ventilation mode that provides pressure assist (or mandatory) breaths and pressure support (or spontaneous) breaths, based on a set of rules. ${ }^{5,14}$ To initiate ASV, the clinician first enters the patient's ideal body weight (IBW), per Devine's formulas ${ }^{15}$ :

$$
\text { Male: } \mathrm{IBW}=50+2.3 \text { (height in inches }-60)
$$

Female: IBW $=45.5+2.3$ (height in inches -60$)$ 
The clinician then sets the \% MinVol, which determines the target $\dot{\mathrm{V}}_{\mathrm{E}}$. The \% MinVol at $100 \%$ will provide a target $\dot{\mathrm{V}}_{\mathrm{E}}$ of $0.1 \mathrm{~L} / \mathrm{min} / \mathrm{kg} \mathrm{IBW}$. At any given $\dot{\mathrm{V}}_{\mathrm{E}}$, ASV constantly looks for a combination of $\mathrm{V}_{\mathrm{T}}$ and respiratory rate that is within the safety boundaries determined by the ASV rules and best satisfies the minimum work of breathing concept of Otis et al. ${ }^{7}$ If no patient effort is detected, the target breathing pattern is imposed with controlled breaths that are pressure-limited, volume-targeted, and time-cycled (pressure controlled breaths). If the patient is making breathing efforts, ASV delivers pressure-limited, volume-targeted, and flow-cycled (ie, pressure support) breaths. As the patient contribution to the target $\dot{\mathrm{V}}_{\mathrm{E}}$ increases, the ASV algorithm automatically reduces the rate of controlled breaths. When all the breaths are triggered by the patient, ASV attempts to achieve an optimal breathing pattern by controlling only the inspiratory pressure, and thus $\mathrm{V}_{\mathrm{T}}$. As the patient recovers, ASV reduces the inspiratory pressure. The lowest inspiratory pressure ASV provides is $5 \mathrm{~cm} \mathrm{H}_{2} \mathrm{O}$.

\section{Ventilator Management}

For the patients managed with non-ASV modes, ventilator changes were made according to a ventilator protocol. After intubation, patients with acute respiratory failure are initially supported with either volume or pressure assist control mode, with a $\mathrm{V}_{\mathrm{T}}$ of $5-7 \mathrm{~mL} / \mathrm{kg}$, to maintain $\mathrm{pH}$ of 7.30-7.45, $\mathrm{P}_{\mathrm{aO}_{2}}$ of 55-80 $\mathrm{mm} \mathrm{Hg}$ (or oxygen saturation of 88-95\%), and plateau pressure of $<33 \mathrm{~cm} \mathrm{H}_{2} \mathrm{O}$. PEEP is adjusted downward when $\mathrm{F}_{\mathrm{IO}_{2}}$ is $\leq 0.6$. When the patients reaches PEEP $\leq 10 \mathrm{~cm} \mathrm{H}_{2} \mathrm{O}, \mathrm{F}_{\mathrm{IO}_{2}} \leq 0.6$, and $\mathrm{pH} \geq 7.30$, partial support modes, including pressure support ventilation (PSV) or PSV/SIMV, are used. The protocol also recommends that for patients whose $\mathrm{P}_{\mathrm{aO}_{2}} / \mathrm{F}_{\mathrm{IO}_{2}}$ is $>200 \mathrm{~mm} \mathrm{Hg}$, PEEP should be $\leq 5 \mathrm{~cm} \mathrm{H}_{2} \mathrm{O}$, with no use of sedatives or vasopressors, and should have a daily 30 min spontaneous breathing trial (SBT) on T-piece, to assess for extubation readiness. For patients managed with $\mathrm{ASV}$, the RT set the initial \% MinVol and recorded the mandatory rate, spontaneous rate, and inspiratory pressure level. If a patient did not have a spontaneous rate on $100 \%$ MinVol, the $\%$ MinVol was decreased to $80 \%$. All the ventilator changes were made by the RT.

Full-time RT coverage was available in the ICU during the day shift (8:00 AM to 5:00 PM). The RT rounds on all ventilated patients twice a day (once in the morning and once in the afternoon). During those 2 visits the RT makes changes to the ventilator setting, and updates the respiratory log with patient demographics; admission date; intubation date; endotracheal tube size; extubation date; clinician-set variables (ventilation mode, respiratory rate, $\mathrm{V}_{\mathrm{T}}$, $\mathrm{F}_{\mathrm{IO}_{2}}$, PEEP); measured variables (respiratory rate, inspiratory-expiratory ratio, oxygen saturation, expiratory $\mathrm{V}_{\mathrm{T}}$, peak airway pressure, $\dot{\mathrm{V}}_{\mathrm{E}}$, compliance, and resistance); SBT results; and bedside mechanics. There is no on-site RT coverage in the evening (from 5:00 PM to 8:00 AM), so ventilator issues are managed by the primary ICU nurse and reported to the on-call attending physician, who is not in the ICU.

\section{Study Protocol}

Upon enrollment into the ASV group, the ventilator setting was changed to $\% \mathrm{MinVol}$ of $100 \%$. If the patient showed some spontaneous breaths, $100 \%$ MinVol was maintained. If there were no spontaneous breaths, the $\% \mathrm{MinVol}$ was decreased to $80 \%$. This starting \% MinVol setting was based on previous trials with post-cardiacsurgery patients. ${ }^{8,10-12}$ If the patient could not tolerate these ASV settings (eg, developed respiratory distress), we switched back to the original ventilator setting and excluded the patient from the study. The control group was managed according to the ventilator protocol described above.

We defined extubation readiness as a frequency/tidalvolume ratio $\left(\mathrm{f} / \mathrm{V}_{\mathrm{T}}\right)$ of $<105$ (breaths $/ \mathrm{min}$ ) $/ \mathrm{L}$ on pressure support of $\leq 8 \mathrm{~cm} \mathrm{H}_{2} \mathrm{O}$ and PEEP of $\leq 5 \mathrm{~cm} \mathrm{H}_{2} \mathrm{O}$ for at least $2 \mathrm{~h}$, and all spontaneous breaths. The SBT consisted of a 30-min T-piece trial, and we extubated if $\mathrm{f} / \mathrm{V}_{\mathrm{T}}$ remained below 105 (breaths/min)/L during the SBT. Both groups received the same standard of care provided by the medical ICU team, which follows the guidelines and protocols established in our ICU, which include lung-protection ventilation strategy, sedation, and extubation criteria. Patients in both groups were evaluated twice a day by the RT.

We recorded baseline clinical characteristics of all patients, including demographics, Acute Physiology and Chronic Health Evaluation II score, ventilator settings, preenrollment ventilator days, causes for intubation, and arterial blood gas values. All patients were followed for at least 3 weeks after enrollment. We defined extubation success as no re-intubation for at least 48 hours. Our primary outcomes were the number of patients who reached the "liberation potential" and the probability of liberation from the ventilator within 3 weeks of enrollment.

\section{Statistical Analysis}

Data are expressed as mean \pm SD or median and IQR, as appropriate. The prospective data from the ASV group and the retrospective data from the non-ASV group were analyzed simultaneously. For differences between the ASV and non-ASV groups we analyzed normally distributed data with the Student $t$ test, and binomially distributed data with the Fisher exact test. We used the Mann-Whitney test for the differences in ICU stay and hospital stay. We con- 

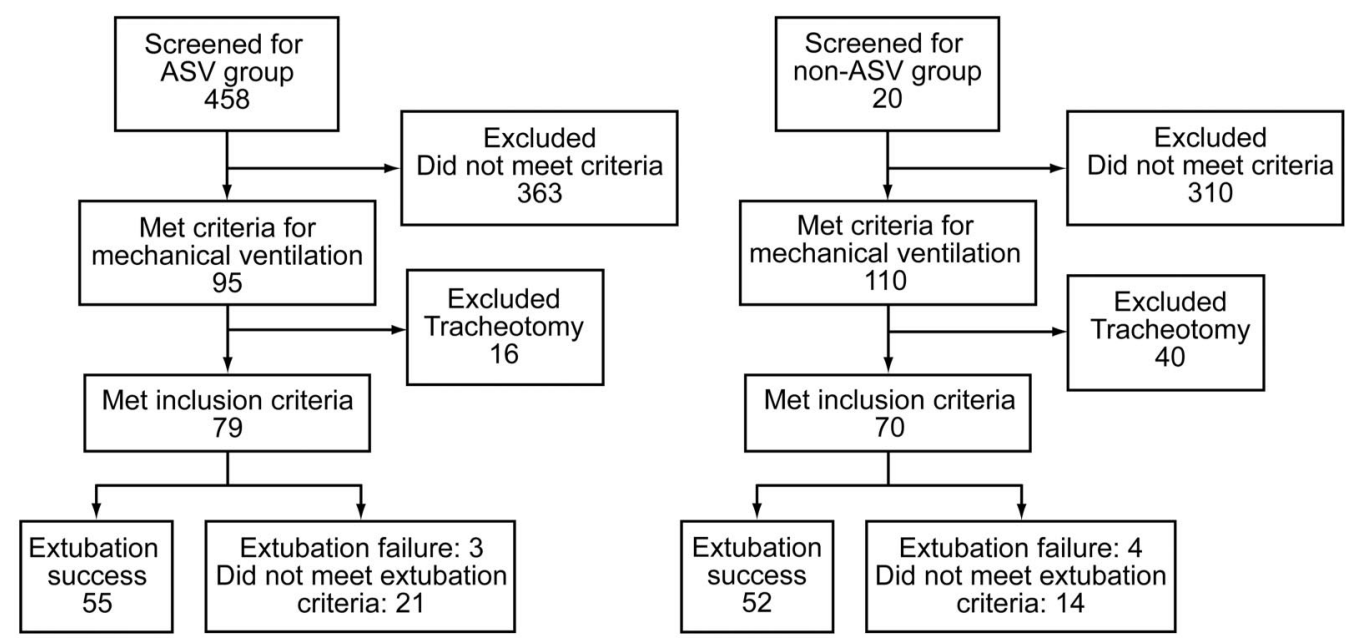

Fig. 1. Flow chart. ASV = adaptive support ventilation.

structed a cumulative incidence plot for the probability of liberation from mechanical ventilation. The difference between the 2 groups was determined with the log-rank test. We calculated the sample size with the number of patients who reached extubation readiness as the outcome. To detect a $20 \%$ difference with a standard deviation of $30 \%$, an alpha of .05 and a $\beta$ of .80 , we needed at least 64 patients in each group. We also performed multiple logistic regression analysis to determine factors that contributed to the differences in time to extubation readiness. The independent variables used in that analysis were group (ASV vs non-ASV); age; sex; admission diagnosis; ventilation mode at enrollment; and use of sedatives, opioids, and inotropes. The analyses were performed with statistics software (SPSS 15.0, SPSS, Chicago, Illinois). A $P$ value of $<.05$ was considered statistically significant.

\section{Results}

We screened 458 patients and enrolled 79 patients in the ASV group (Fig. 1). The reasons for exclusion were persistent hemodynamic instability, consent refused, attending physician refused, attending physicians indicated a high likelihood of death within 6 weeks, and tracheostomy. Extubation was attempted in 58 patients, and 55 patients were successfully extubated. Three patients $(5 \%)$ were reintubated within 48 hours. The remaining 21 patients never reached extubation readiness and were considered weaning failures. For the non-ASV group, we screened 420 patient records, of which 70 met the inclusion criteria (see Fig. 1). The reasons for exclusion were similar to those in the ASV group, and included persistent hemodynamic instability, probable death within 6 weeks, not on a Galileo ventilator, and tracheostomy. In the non-ASV group extubation was attempted in 56 patients, and 52 patients were successfully extubated. Four patients $(7 \%)$ were re-intubated within 48 hours. The remaining 14 patients were considered weaning failures. The clinical characteristics were similar between the 2 groups (Table 1).

Overall, extubation was attempted in $73 \%$ in the ASV group and $80 \%$ in the non-ASV group $(P=.45$ via chisquare test). In the ASV group, 20\% achieved extubation readiness within 1 day of enrollment, versus $4 \%$ in the non-ASV group $(P<.001$ via the Fisher exact test) (Fig. 2$)$. In the non-ASV group the recognition of extubation readiness tended to be delayed. The median time from enrollment to extubation readiness tended to be shorter in the ASV group: one day in the ASV group vs 3 days in the non-ASV group $(P=.055)$ (Table 2$)$. The cumulative incidence plot (Fig. 3) shows that patients in the ASV group were more likely to reach extubation readiness in a shorter time and more likely to be liberated from mechanical ventilation within 3 weeks of enrollment $(P=.04)$ (Fig. 4). There were no significant differences in ICU or hospital stay. Multiple logistic regression analysis showed that, of the independent factors, only group (ASV vs nonASV) was associated with shorter time to extubation readiness $(P=.048$ via the likelihood ratio test).

\section{Discussion}

One of the theoretical advantages of ASV is to facilitate weaning because it automatically adjusts the pressure support level (inspiratory pressure) as the patient's lung condition improves. To date, ASV has been shown to shorten the time to extubation in patients who underwent cardiac surgery ${ }^{8-12}$ and who did not have substantial lung disease. They were ventilated primarily for anesthesia and could be extubated quickly after surgery. Thus, those results may not be applicable to patients who were ventilated for acute 
Table 1. Baseline Characteristics

\begin{tabular}{|c|c|c|c|}
\hline & $\begin{array}{c}\text { ASV } \\
(n=79)\end{array}$ & $\begin{array}{l}\text { Usual Care } \\
(n=70)\end{array}$ & $P$ \\
\hline Age (y) & $72.9 \pm 16.0$ & $72.2 \pm 14.5$ & .80 \\
\hline Male/female & $46 / 33$ & $48 / 39$ & .13 \\
\hline APACHE II score at admission & $23.8 \pm 6.8$ & $23.8 \pm 7.7$ & .97 \\
\hline \multicolumn{3}{|l|}{ Admission Diagnosis, no. (\%) } & .06 \\
\hline Pneumonia & $49(62)$ & $29(41)$ & \\
\hline COPD & $5(6)$ & $9(13)$ & \\
\hline Other pulmonary diseases & $1(1)$ & $5(7)$ & \\
\hline Sepsis & $4(5)$ & $7(10)$ & \\
\hline Cancer & $5(6)$ & $8(11)$ & \\
\hline Congestive heart failure & $0(0)$ & $1(1)$ & \\
\hline Upper gastrointestinal bleeding & $1(1)$ & $3(4)$ & \\
\hline End-stage renal disease & $0(0)$ & $1(1)$ & \\
\hline Other & $14(18)$ & $7(10)$ & \\
\hline Mechanical ventilation before enrollment (d) & $3.8 \pm 3.3$ & $3.3 \pm 3.7$ & .41 \\
\hline PS/SIMV mode, no. (\%) & $64(81)$ & $25(36)$ & .01 \\
\hline PS level $\left(\mathrm{cm} \mathrm{H}_{2} \mathrm{O}\right)$ & $16.7 \pm 2.9$ & $16.7 \pm 3.1$ & .97 \\
\hline Minute volume (L/min) & $8.7 \pm 2.9$ & $9.1 \pm 3.0$ & .42 \\
\hline Respiratory rate (breaths/min) & $19 \pm 5$ & $19 \pm 5$ & .99 \\
\hline Tidal volume (mL) & $451 \pm 110$ & $457 \pm 104$ & .74 \\
\hline $\mathrm{S}_{\mathrm{pO}_{2}}(\%)$ & $99 \pm 2$ & $98 \pm 2$ & .01 \\
\hline $\mathrm{P}_{\mathrm{aO}_{2}} / \mathrm{F}_{\mathrm{IO}_{2}}(\mathrm{~mm} \mathrm{Hg})$ & $313 \pm 102$ & $310 \pm 121$ & .86 \\
\hline $\mathrm{P}_{\mathrm{aCO}_{2}}(\mathrm{~mm} \mathrm{Hg})$ & $35 \pm 8$ & $33 \pm 9$ & .22 \\
\hline Sedatives (midazolam or propofol), no. (\%) & $23(29)$ & $32(46)$ & .06 \\
\hline Opioids (morphine or meperidine), no. (\%) & $0(0)$ & $3(4)$ & .10 \\
\hline Inotropes (dopamine, noradrenaline, and vasopressin), no. (\%) & $24(30)$ & $34(49)$ & .06 \\
\hline Albumin $(\mathrm{gm} / \mathrm{dL})$ & $2.6 \pm 0.6$ & $2.7 \pm 0.5$ & .14 \\
\hline $\begin{array}{l} \pm \text { values are mean } \pm \text { SD. } \\
\text { ASV }=\text { adaptive support ventilation } \\
\text { APACHE = Acute Physiology and Chronic Health Evaluation } \\
\text { PS = pressure support } \\
\text { SIMV = synchronized intermittent mandatory ventilation }\end{array}$ & & & \\
\hline
\end{tabular}

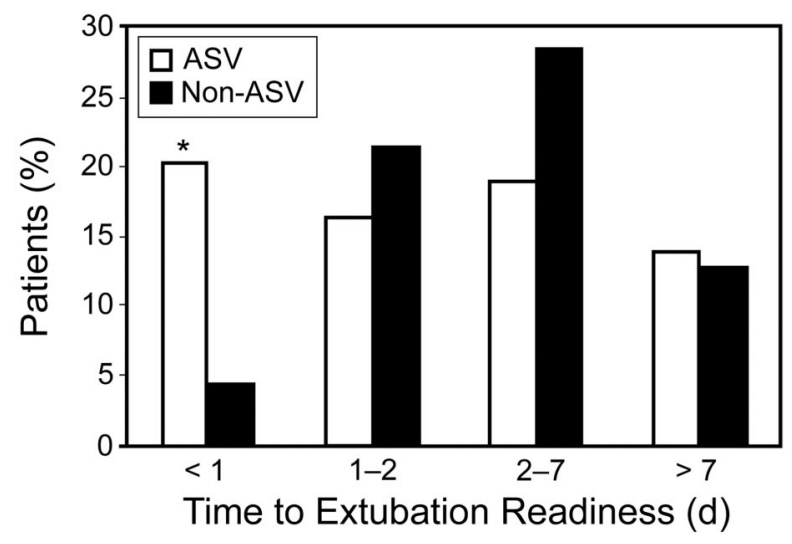

Fig. 2. Time to extubation readiness after enrollment. Approximately $20 \%$ of patients in the adaptive support ventilation (ASV) group achieved extubation readiness within one day of enrollment. ${ }^{*} P<.001$ versus the non-ASV group, via Fisher's exact test.

respiratory failure. In the present pilot study we selected patients recovering from acute respiratory failure on PSV
Table 2. Clinical Outcomes of Extubated Patients

\begin{tabular}{lccc}
\hline \hline & \multicolumn{2}{c}{$\begin{array}{c}\text { Median and } \\
\text { Interquartile Range (d) }\end{array}$} & \\
\cline { 2 - 3 } & $\begin{array}{c}\text { ASV } \\
(n=58)\end{array}$ & $\begin{array}{c}\text { Non-ASV } \\
(n=56)\end{array}$ & $P$ \\
\hline Time to extubation readiness* & $1(1-4)$ & $3(1-5)$ & .055 \\
ICU stay & $9(6-15)$ & $8.5(6-13)$ & .43 \\
Hospital stay & $34(18-50)$ & $36(21-53)$ & .27 \\
& & & \\
$*$ Time to extubation readiness was the time from enrollment to achieving a ratio of & \\
respiratory frequency to tidal volume of $<105$ (breaths/min)/L on pressure support of & \\
$\leq 8$ cm $\mathrm{H}_{2} \mathrm{O}$ for at least 2 hours & & & \\
ASV $=$ adaptive support ventilation. & & \\
ICU $=$ intensive care unit & & \\
\hline
\end{tabular}

or PSV/SIMV and managed with ASV. One major finding in our study was that $20 \%$ of the patients achieved extubation readiness within one day of enrollment (ie, after switching to ASV). In the non-ASV group, that number was $4 \%$. Overall, extubation readiness was recognized ear- 


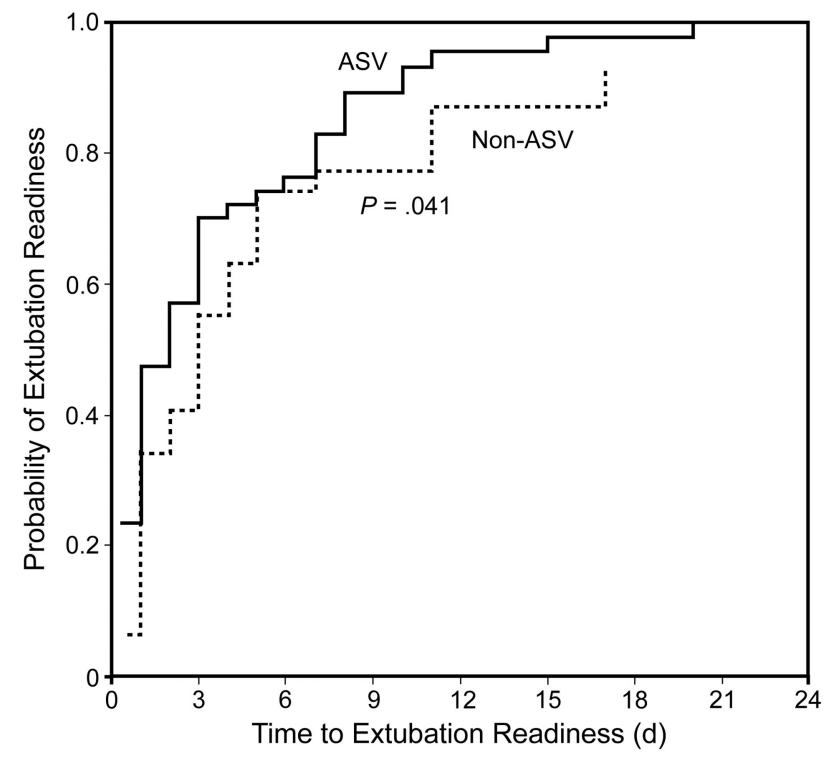

Fig. 3. Cumulative incidence of the probability of reaching extubation readiness (see text) after enrollment.

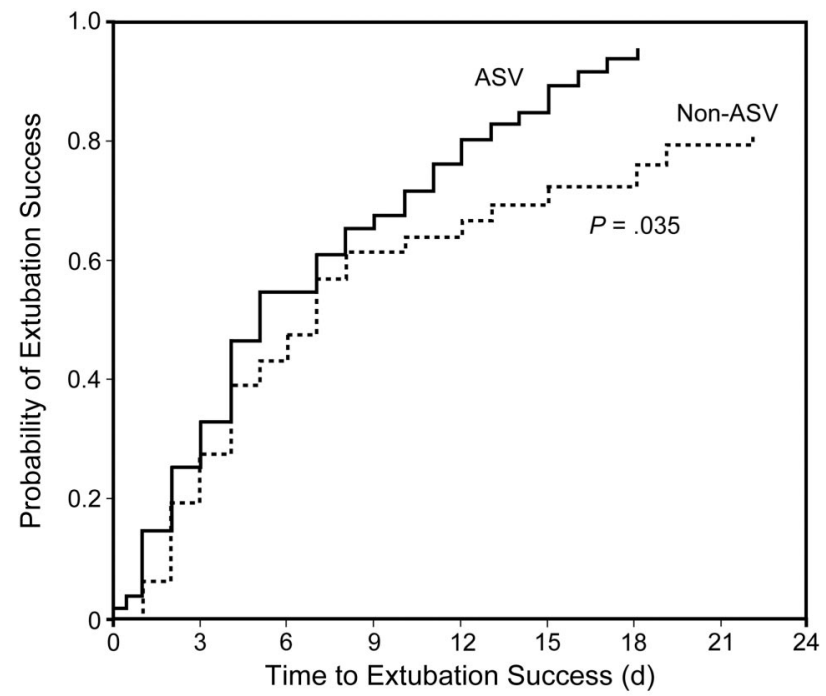

Fig. 4. Cumulative incidence of the probability of extubation success (see text) after enrollment.

lier with ASV, leading to a greater likelihood of liberation from mechanical ventilation. Since SIMV mode is still commonly used in ICUs throughout the world, ${ }^{16}$ our results should be applicable to many ICUs similar to ours.

Extubation readiness requires frequent assessment of the patient's respiratory status. Failure to recognize extubation readiness in a timely manner delays ventilator liberation and may increase morbidity and mortality associated with mechanical ventilation. The prevalence of such delay is unknown and is probably dependent on system factors, including staffing, training, and patient population. We found that extubation readiness may not be rec- ognized in at least $15 \%$ of the patients recovering from respiratory failure in an ICU with a low RT/patient ratio. Strategies such as mandatory daily SBT can help detect extubation readiness. ${ }^{17-20}$ SBT requires close monitoring, especially during the early phase, since unnecessary prolongation of a failing SBT may cause muscle overload. Therefore, when the ICU work load is high relative to available manpower, SBT, which is considered non-urgent, tends to be delayed. Under these circumstances an automated ventilation system such as ASV can help recognize extubation readiness with fewer manipulations. ${ }^{9}$ Another computerized system, SmartCare/PS, also detected extubation readiness earlier than the intensivists in a group of patients on prolonged mechanical ventilation. ${ }^{21}$

Various computerized algorithms have been used to assist weaning, and most of those studies showed promise in shortening time to extubation..$^{9,10,12,22-24}$ But the superior results with computerized algorithms in those studies appeared to be due in part to slower weaning in the control group, dictated by the study protocol. In clinical practice slower weaning can be due to system factors such as adequacy of staffing relative to clinical work load and the staff training and experience. While a ventilator protocol helps, effective execution of the protocol is affected by these system factors. For example, weaning tends not to proceed during the evening shift, especially if a full-time RT is not available. An RT who is overwhelmed by managing acute patients tends to delay weaning, which is considered "routine work." An automated ventilator system such as ASV that essentially operates a built-in ventilator protocol continuously may help in this situation. It would be of interest to assess the performance of ASV in ICUs with different system factors. Recently, the reduction in weaning duration with SmartCare/PS shown in a previous study ${ }^{23}$ was not confirmed when patients managed with the automated system were compared to a control group whose weaning was managed by an experienced critical care specialty nurse in an ICU with a 1:1 nurse-to-patient ratio and 24/7 intensivist coverage. ${ }^{24}$ That high level of staffing, however, is usually not achievable in many ICUs.

\section{Limitations}

Our study was not a randomized controlled trial. The data from the ASV group was obtained prospectively, but the non-ASV group data were obtained retrospectively. To minimize the bias, we obtained the non-ASV group data from the period immediately before the ASV study period, and data was collected by an RT who was unaware of the study hypothesis and outcome measures. The physician, nursing, and RT staffing levels were the same during the control and ASV study periods, and the fact that the study periods were adjacent minimized the risk of changes in clinical practice. The 2 groups had similar pre-enrollment 


\section{Adaptive Support Ventilation in a Medical Intensive Care Unit}

clinical characteristics. The successful extubation rates were similar: $70 \%$ for the ASV group and $74 \%$ for the non-ASV group. The re-intubation rates were also similar (5\% for the ASV group and 7\% for the non-ASV group). These rates are similar to those in previous weaning trials. ${ }^{23,25-27}$ The control group's median ventilator days ( $\left.10 \mathrm{~d}\right)$ was also the same as that of the entire medical ICU population in 2007 (10 d), indicating that the ventilator care of the control patients was similar to that of the entire medical ICU patient population in our center (data not shown). In addition, both sets of data were analyzed simultaneously, so the ASV group's outcomes were not known when the non-ASV group's data were being collected. Our study was not designed and powered to investigate other ICU outcomes such as ventilator-associated pneumonia, use of sedation, or ICU stay, that may be influenced by mechanical ventilation time. In the ASV group we used $80-$ $100 \%$ MinVol to standardize the protocol, but that setting may still be too high for some patients and may prevent the decrease of inspiratory pressure. Unless the \% MinVol is further adjusted downward, extubation readiness may not be recognized.

\section{Conclusions}

Extubation readiness was not recognized in a timely manner in at least $15 \%$ of the patients recovering from respiratory failure, even in the presence of a ventilator protocol. ASV can help identify these patients and may improve weaning outcomes. These results support the need for future randomized controlled trials.

\section{ACKNOWLEDGMENT}

We thank Ching-Huang Lai MD, School of Public Health of the National Defense Medical Center, Taipei, Taiwan, for statistical advice and consultation.

\section{REFERENCES}

1. Burns KE, Lellouche F, Lessard MR. Automating the weaning process with advanced closed-loop systems. Intensive Care Med 2008; 34(10): 1757-1765.

2. Dojat M, Harf A, Touchard D, Lemaire F, Brochard L. Clinical evaluation of a computer-controlled pressure support mode. Am J Respir Crit Care Med 2000;161(4 Pt 1):1161-1166.

3. Dojat M, Harf A, Touchard D, Laforest M, Lemaire F, Brochard L. Evaluation of a knowledge-based system providing ventilatory management and decision for extubation. Am J Respir Crit Care Med 1996;153(3):997-1004.

4. Brunner JX. Principles and history of closed-loop controlled ventilation. Respir Care Clin N Am 2001;7(3):341-362.

5. Brunner JX, Iotti GA. Adaptive support ventilation (ASV). Minerva Anestesiol 2002;68(5):365-368.

6. Campbell RS, Branson RD, Johannigman JA. Adaptive support ventilation. Respir Care Clin N Am 2001;7(3):425-440.
7. Otis AB, McKerrow CB, Bartlett RA, Mead J, Mcllroy MB, SelverStone NJ, et al. Mechanical factors in distribution of pulmonary ventilation. J Appl Physiol 1956;8(4):427-443.

8. Gruber PC, Gomersall CD, Leung P, Joynt GM, Ng SK, Ho KM, et al. Randomized controlled trial comparing adaptive-support ventilation with pressure-regulated volume-controlled ventilation with automode in weaning patients after cardiac surgery. Anesthesiology 2008;109(1):81-87.

9. Petter AH, Chiolero RL, Cassina T, Chassot PG, Muller XM, Revelly JP. Automatic "respirator/weaning" with adaptive support ventilation: the effect on duration of endotracheal intubation and patient management. Anesth Analg 2003;97(6):1743-1750.

10. Sulzer CF, Chiolero R, Chassot PG, Mueller XM, Revelly JP. Adaptive support ventilation for fast tracheal extubation after cardiac surgery: a randomized controlled study. Anesthesiology 2001;95(6): 1339-1345.

11. Dongelmans DA, Veelo DP, Paulus F, de Mol BA, Korevaar JC, Kudoga A, et al. Weaning automation with adaptive support ventilation: a randomized controlled trial in cardiothoracic surgery patients. Anesth Analg 2009;108(2):565-571.

12. Cassina T, Chiolero R, Mauri R, Revelly JP. Clinical experience with adaptive support ventilation for fast-track cardiac surgery. J Cardiothorac Vasc Anesth 2003;17(5):571-575.

13. Tassaux D, Dalmas E, Gratadour P, Jolliet P. Patient-ventilator interactions during partial ventilatory support: a preliminary study comparing the effects of adaptive support ventilation with synchronized intermittent mandatory ventilation plus inspiratory pressure support. Crit Care Med 2002;30(4):801-807.

14. Iotti GA, Brunner JX, Braschi A, Laubscher T, Olivei MC, Palo A, et al. Closed-loop control of airway occlusion pressure at 0.1 second (P0.1) applied to pressure-support ventilation: algorithm and application in intubated patients. Crit Care Med 1996;24(5):771-779.

15. Devine BJ. Gentamicin therapy. Drug Intell Clin Pharm 1974;8:650655.

16. Ortiz G, Frutos-Vivar F, Ferguson ND, Esteban A, Raymondos K, Apezteguia $\mathrm{C}$, et al. Outcomes of patients ventilated with synchronized intermittent mandatory ventilation with pressure support: a comparative propensity score study. Chest;137(6):1265-1277.

17. Robertson TE, Sona C, Schallom L, Buckles M, Cracchiolo L, Schuerer D, et al. Improved extubation rates and earlier liberation from mechanical ventilation with implementation of a daily spontaneous-breathing trial protocol. J Am Coll Surg 2008;206(3):489-495.

18. Kamlin CO, Davis PG, Argus B, Mills B, Morley CJ. A trial of spontaneous breathing to determine the readiness for extubation in very low birth weight infants: a prospective evaluation. Arch Dis Child Fetal Neonatal Ed 2008;93(4):F305-306.

19. Newth CJ, Venkataraman S, Willson DF, Meert KL, Harrison R, Dean JM, et al. Weaning and extubation readiness in pediatric patients. Pediatr Crit Care Med 2009;10(1):1-11.

20. MacIntyre NR, Cook DJ, Ely EW Jr, Epstein SK, Fink JB, Heffner $\mathrm{JE}$, et al. Evidence-based guidelines for weaning and discontinuing ventilatory support: a collective task force facilitated by the American College of Chest Physicians; the American Association for Respiratory Care; and the American College of Critical Care Medicine. Chest 2001;120(6 Suppl):375S-395S.

21. Bouadma L, Lellouche F, Cabello B, Taille S, Mancebo J, Dojat M, et al. Computer-driven management of prolonged mechanical ventilation and weaning: a pilot study. Intensive Care Med 2005;31(10): 1446-1450.

22. Hendrix H, Kaiser ME, Yusen RD, Merk J. A randomized trial of automated versus conventional protocol-driven weaning from mechanical ventilation following coronary artery bypass surgery. Eur J Cardiothorac Surg 2006;29(6):957-963. 


\section{Adaptive Support Ventilation in a Medical Intensive Care Unit}

23. Lellouche F, Mancebo J, Jolliet P, Roeseler J, Schortgen F, Dojat M, et al. A multicenter randomized trial of computer-driven protocolized weaning from mechanical ventilation. Am J Respir Crit Care Med 2006;174(8):894-900.

24. Rose L, Presneill JJ, Johnston L, Cade JF. A randomised, controlled trial of conventional versus automated weaning from mechanical ventilation using SmartCare/PS. Intensive Care Med 2008;34(10): 1788-1795

25. Esteban A, Frutos F, Tobin MJ, Alia I, Solsona JF, Valverdu I, et al. A comparison of four methods of weaning patients from mechanical ventilation. Spanish Lung Failure Collaborative Group. N Engl J Med 1995;332(6):345-350.

26. Esteban A, Alia I, Tobin MJ, Gil A, Gordo F, Vallverdu I, et al. Effect of spontaneous breathing trial duration on outcome of attempts to discontinue mechanical ventilation. Spanish Lung Failure Collaborative Group. Am J Respir Crit Care Med 1999;159(2):512-518.

27. Perren A, Domenighetti G, Mauri S, Genini F, Vizzardi N. Protocoldirected weaning from mechanical ventilation: clinical outcome in patients randomized for a 30-min or 120-min trial with pressure support ventilation. Intensive Care Med 2002;28(8):1058-1063. 